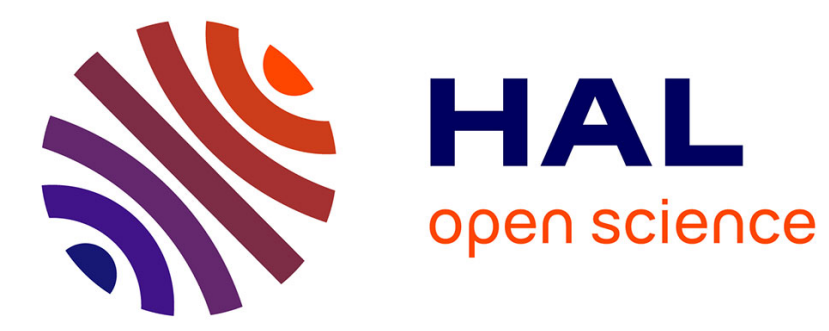

\title{
Kinematic Modeling and Singularity Treatment of Steerable Wheeled Mobile Robots with Joint Acceleration Limits
}

Mohamed Sorour, Andrea Cherubini, Robin Passama, Philippe Fraisse

\section{To cite this version:}

Mohamed Sorour, Andrea Cherubini, Robin Passama, Philippe Fraisse. Kinematic Modeling and Singularity Treatment of Steerable Wheeled Mobile Robots with Joint Acceleration Limits. ICRA: International Conference on Robotics and Automation, May 2016, Stockholm, Sweden. pp.2110-2115, 10.1109/ICRA.2016.7487360 . hal-01274772

\section{HAL Id: hal-01274772}

\section{https://hal.science/hal-01274772}

Submitted on 17 Feb 2016

HAL is a multi-disciplinary open access archive for the deposit and dissemination of scientific research documents, whether they are published or not. The documents may come from teaching and research institutions in France or abroad, or from public or private research centers.
L'archive ouverte pluridisciplinaire HAL, est destinée au dépôt et à la diffusion de documents scientifiques de niveau recherche, publiés ou non, émanant des établissements d'enseignement et de recherche français ou étrangers, des laboratoires publics ou privés. 


\title{
Kinematic Modeling and Singularity Treatment of Steerable Wheeled Mobile Robots with Joint Acceleration Limits
}

\author{
Mohamed Sorour ${ }^{1,2}$, Andrea Cherubini ${ }^{1}$, Robin Passama ${ }^{1}$, and Philippe Fraisse ${ }^{1}$
}

\begin{abstract}
Non-holonomic omnidirectional mobile robots have higher load carrying capacity than their holonomic counterparts. Once the steer joint configuration is initialized, they can perform arbitrarily complex three-dimensional trajectories in the plane of motion and, as such, are more suitable for industrial contexts. However, their kinematic model presents representational and structural singularities, solutions to which must respect actuator performance limits. Recent research efforts have provided either simple restricting of the velocity space (among which few considered hardware limits) or complex non-restricting (no hardware limits considered) solutions. Most of these efforts are providing solutions at the kinematic control level. Instead, here we propose both a representational singularity free kinematic model, and a simple numeric treatment for the kinematic singularity. We further provide a method to tune the latter, to respect the actuator acceleration limits. Thanks to its steer rate damping behavior, the method can be further extended, to respect joint limits. Another benefit is the treatment of the singularity at the level of the kinematic model, which enhances real time capabilities. The developed method has been tested successfully on the Neobotix-MPO700 mobile robot and shown superior results as compared to the embedded controller.

Index Terms - Wheeled mobile robots, Steerable wheels.
\end{abstract}

\section{INTRODUCTION}

Mobile robots equipped with fully steerable wheels are usually described as pseudo-omnidirectional (or nonholonomic or quasi onmi), providing a distinction from the omnidirectional ones, that employ either castor or omnidirectional (swedish) wheels. Since steerable wheels are usually characterized by higher load carrying capacity than castor or omni-wheels, pseudo-omni platforms are more adequate for industrial applications. Although these platforms possess only one degree of mobility (i.e., the directly controllable degree of freedom, here the rotation about the instantaneous center of rotation - ICR), they can perform complex three-dimensional trajectories in the plane, only after reorienting the steer joints to the proper initial conditions. Thus, they do not require special maneuvers, and are more suitable than less complex systems (e.g., differential drive, car) for limited footprint applications.

Kinematic modeling of steerable (centered or offcentered) mobile robots is usually done using the Cartesian coordinates of the ICR [1], the polar coordinates of the ICR [2-4], or by considering the Cartesian coordinates of the robot frame $[5,6]$. Each of these methods has its own mathematical singularities, indicated in the literature as representational

\footnotetext{
1 Interactive Digital Human group IDH, Laboratory for Computer Science, Micro-electronics and Robotics LIRMM - University of Montpellier CNRS, 860 rue de Saint Priest, 34090 Montpellier, France. first. lastnamedirmm. fr.

${ }^{2}$ PSA Peugeot-Citroen, Velizy Villacoublay, France.
}

singularities. This type of singularity results from two distinct situations, namely having null angular and/or linear velocity. Employing the Cartesian coordinates is less prone to such singularities, especially if 3 or more wheels are used [5].

Kinematic (or structural) singularity also exists. This refers to cases where the ICR passes by (or nearby) any of the steering axes. Then, the steer angle is not uniquely defined (infinite solutions respect the kinematic constraints). In the neighborhood of such singularity, the steering rate grows unbounded. Different approaches have been employed to deal with structural singularities. The admissible velocity space has been constrained in [3]. Repulsive potential fields have been constructed at the steering axes in [1, 4, 7]. Although such techniques reduce the available velocity space, they suffice in static environments. However, in the emerging industrial applications involving human robot collaboration, the environment can change suddenly, due to moving operators. Then, all the available velocity workspace should be exploited, including worst-case scenarios with 1) ICR passing by and/or 2) ICR stopping at a steering axis (kinematic singularity). Besides, the controller must respect joint acceleration limits and mechanical constraints $[4,8,9]$.

Such issues have recently drawn researchers' attention. In [10], a complex formulation provides a locally singular-free representation, by switching between several state space representations of the twist. In [11], a time optimal controller is developed to account for the joint velocity limits. Such controller is shown to be successful in passing by singular configurations, while respecting the actuator velocity limits. Acceleration limits were further taken into account in [8]. However, the methods developed in [11] and [8] result in the maximum steering/driving velocity for the axis passed by the ICR. While they can handle the scenario 1 mentioned above, they are not suitable for scenario 2. The simulations in [10] do not provide information on joint axes rates in the vicinity and at the singular configurations.

In this work, we propose a simple, yet numerically robust, treatment of the kinematic model. Such treatment introduces a damping effect, to oppose the singular behavior. Consequently, it solves not only the aforementioned 2 worstcase scenarios, but also provides a promising candidate to the joint limits issue encountered in [4]. We provide a formula for online tuning of the damping effect, which maintains the joint accelerations below their limits. Additionally, we propose a benchmark test trajectory for evaluating, at all singular conditions, the performance of novel kinematic controllers for this class of mobile robots.

This paper is organized as follows: Section II presents the forward and inverse kinematic models, along with the joint initialization strategy. Section III and IV respectively detail the proposed treatment of kinematic singularities, and the benchmark testing scenario. The experimental results are provided in section $\mathrm{V}$, and we conclude in section VI. 


\section{KINEMATIC MODEL}

The kinematic model developed in this section is inspired by the pioneering work of Muir et al. [12], Campion et al. [6], [12] and Low et al. [14]. In Fig. 1, let $\boldsymbol{R}_{I}=$ $\left(o_{I}, \boldsymbol{x}_{I}, \boldsymbol{y}_{I}, \boldsymbol{z}_{I}\right)$ be the inertial (world) reference frame, $\boldsymbol{R}_{b}=\left(o_{b}, \boldsymbol{x}_{b}, \boldsymbol{y}_{b}, \boldsymbol{z}_{b}\right)$ the mobile base attached frame with the origin $o_{b}$ located at its geometric center, $\boldsymbol{R}_{h i}=$ $\left(o_{h i}, \boldsymbol{x}_{h i}, \boldsymbol{y}_{h i}, \boldsymbol{z}_{h i}\right)$ is the $i^{\text {th }}$ hip frame, where $i=1, \ldots, 4$; a frame attached to the fixed part of the steering joint (related to the base frame by a fixed transformation matrix) whereas the steering frame $\boldsymbol{R}_{s i}=\left(o_{s i}, \boldsymbol{x}_{s i}, \boldsymbol{y}_{s i}, \boldsymbol{z}_{s i}\right)$ is attached to the movable part. The hip and steering frames share the same origin, with relative orientation depending on the steering angle $\beta_{i}, \boldsymbol{R}_{w i}=\left(o_{w i}, \boldsymbol{x}_{w i}, \boldsymbol{y}_{w i}, \boldsymbol{z}_{w i}\right)$ is a frame attached to (but doesn't rotate with) $i^{\text {th }}$ wheel. Frame $\boldsymbol{\mathcal { R }}_{w i}$ is assigned such that $\boldsymbol{x}_{w i}$ points along the heading of the wheel, which rotates about $\boldsymbol{y}_{w i}$ by the driving angle $\varphi_{i}$. For all the described frames, the $\boldsymbol{z}$ axis is always pointing upwards. Let the mobile base pose with respect to the inertial frame, expressed in the inertial frame define the task space coordinates and be described by the $3 \times 1$ vector: ${ }^{I} \xi_{b}^{I}=$ $\left[\begin{array}{lll}x & y & \theta\end{array}\right]^{\mathrm{T}}$, to simplify notation, the right sub, superscripts will be omitted in the sequel since they will never change. Let the mobile base joint actuation coordinates be the $8 \times 1$ vector: $\boldsymbol{A}_{b}=\left[\begin{array}{llllll}\beta_{1} & \ldots & \beta_{4} & \varphi_{1} & \ldots & \varphi_{4}\end{array}\right]^{\mathrm{T}}$. Let the wheel velocity at the ground contact point $o_{c}$ be the vector $\boldsymbol{v}_{c i}=$ $\left[\begin{array}{lll}v_{t i} & v_{n i} & 0\end{array}\right]^{\mathrm{T}}$ with $v_{t i}$ and $v_{n i}$ respectively the $i^{t h}$ wheel's tangential and normal velocities:

$$
\begin{gathered}
\boldsymbol{v}_{c i}=\boldsymbol{R}_{b}^{w i}\left[\boldsymbol{R}_{I}^{b} \dot{\boldsymbol{\xi}}+\dot{\theta} \widehat{\boldsymbol{k}}_{b} \times{ }^{b} \overrightarrow{o_{b} o_{c}}+\dot{\beta}_{i} \boldsymbol{R}_{s i}^{b} \widehat{\boldsymbol{k}}_{s i} \times{ }^{s i} \overrightarrow{o_{s l} o_{c}}\right] \\
+\dot{\varphi}_{i} \hat{\boldsymbol{\jmath}}_{w i} \times{ }^{w i} \overrightarrow{o_{w l} o_{c}} .
\end{gathered}
$$

In this equation, $\widehat{\boldsymbol{k}}_{b}, \widehat{\boldsymbol{k}}_{s i}$ and $\hat{\boldsymbol{\jmath}}_{w i}$ are unit vectors along the axes $\boldsymbol{z}_{b}, \boldsymbol{z}_{s i}$ and $\boldsymbol{y}_{w i}$ respectively, $\boldsymbol{R}_{b}^{w i}=\boldsymbol{R}_{b}^{s i}=\operatorname{rot}\left(\boldsymbol{z}_{b},-\beta_{i}\right)$ and $\boldsymbol{R}_{I}^{b}=\operatorname{rot}\left(\boldsymbol{z}_{I},-\theta\right)$ are $3 \times 3$ rotation matrices, the vectors $b \overrightarrow{O_{b} O_{c}},{ }^{s i} \overrightarrow{O_{s l} O_{c}}$ and ${ }^{w i} \overrightarrow{O_{w l} O_{c}}$ (colored red in Fig. 1) are defined with respect to frames $\boldsymbol{R}_{b}, \boldsymbol{R}_{s i}$ and $\boldsymbol{R}_{w i}$ respectively, $h_{x i}= \pm b$ and $h_{y i}= \pm a$ denote the position of the $i^{\text {th }}$ hip frame origin $o_{h i}$ in the base frame $\mathcal{R}_{b}$, while $d$ and $r_{w}$ denote the steerable wheel offset and radius respectively. Using (1) we get the contact point velocities as:

$$
\begin{gathered}
v_{t i}=\left[\begin{array}{lll}
c\left(\beta_{i}\right) & s\left(\beta_{i}\right) & d-h_{y i} c\left(\beta_{i}\right)+h_{x i} s\left(\beta_{i}\right)
\end{array}\right]^{b} \dot{\xi} \\
+d \dot{\beta}_{i}-r_{w} \dot{\varphi}_{i},
\end{gathered}
$$

with ${ }^{b} \dot{\boldsymbol{\xi}}=\boldsymbol{R}_{I}^{b} \dot{\boldsymbol{\xi}}, c(*)$ and $s(*)$ shorthand the trigonometric cosine and sine functions respectively. By setting $v_{t i}=0$ and $v_{n i}=0,(2)$ and (3) respectively describe the rolling without slipping, and the no lateral skidding kinematic constraints. The no-skid constraint restricts the mobile base motion (the wheel cannot move sideways) and forces the existence of a unique ICR, around which all the wheels and the base frame rotate. From (3), we derive the steering angles that respect such constraint:

$$
\beta_{i}=\tan ^{-1}\left(\frac{{ }^{b} \dot{y}+h_{x i} \dot{\theta}}{{ }^{b} \dot{x}-h_{y i} \dot{\theta}}\right) .
$$

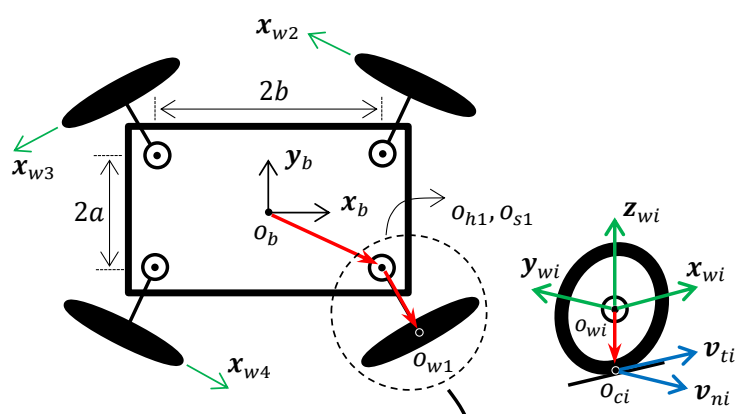

$$
\underset{o_{I}}{\stackrel{y_{I}}{\longrightarrow}} x_{I}
$$

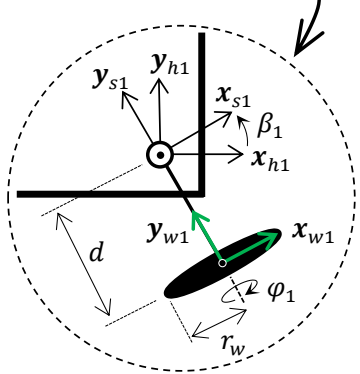

Fig. 1 Modeling schematic for the four fully motorized off-centered, steerable wheeled mobile base

This can be time differentiated to obtain the steering rates:

$$
\dot{\beta}_{i}=\frac{\partial \beta_{i}}{\partial^{b} \dot{x}} b^{\ddot{x}}+\frac{\partial \beta_{i}}{\partial^{b} \dot{y}} b \ddot{y}+\frac{\partial \beta_{i}}{\partial \dot{\theta}} \ddot{\theta}=\boldsymbol{f}_{1 i}\left({ }^{b} \dot{\xi}\right)^{b} \ddot{\boldsymbol{\xi}}
$$

The wheel drive rates can be evaluated using (2) and (5):

$$
\begin{gathered}
\dot{\varphi}_{i}=\frac{1}{r_{w}}\left(\left[c\left(\beta_{i}\right) s\left(\beta_{i}\right) d-h_{y i} c\left(\beta_{i}\right)+h_{x i} s\left(\beta_{i}\right)\right]^{b \dot{\xi}}+d \dot{\beta}_{i}\right) \\
=\frac{1}{r_{w}} \boldsymbol{f}_{2 i}(\boldsymbol{\beta})^{b} \dot{\xi}+\frac{d}{r_{w}} \boldsymbol{f}_{1 i}\left({ }^{b} \dot{\xi}\right)^{b} \ddot{\xi} .
\end{gathered}
$$

In (5) and (6), the steering and wheel drive rates are related to the base velocity ${ }^{b} \dot{\xi}$ and acceleration ${ }^{b} \ddot{\xi}$, expressed in the base frame. These equations can be grouped in matrix form to derive the inverse kinematic actuation model (IKAM):

$$
\begin{aligned}
& \dot{\boldsymbol{A}}_{b}=\boldsymbol{M}_{I}\left({ }^{b} \dot{\xi}\right){ }^{b} \dot{\boldsymbol{\eta}}, \\
& \boldsymbol{M}_{I}\left({ }^{b} \dot{\boldsymbol{\xi}}\right)=\left[\begin{array}{cc}
\mathbf{0}_{4 \times 3} & \boldsymbol{F}_{1}\left({ }^{b} \dot{\boldsymbol{\xi}}\right) \\
\frac{1}{r_{w}} \boldsymbol{F}_{2}(\boldsymbol{\beta}) & \frac{d}{r_{w}} \boldsymbol{F}_{1}\left({ }^{b} \dot{\boldsymbol{\xi}}\right)
\end{array}\right], \\
& \boldsymbol{F}_{1}(b \dot{\xi})=\left[\begin{array}{lll}
\boldsymbol{f}_{11}^{T} & \ldots & \boldsymbol{f}_{14}^{T}
\end{array}\right]^{\mathrm{T}}, \\
& \boldsymbol{F}_{2}(\boldsymbol{\beta})=\left[\begin{array}{lll}
\boldsymbol{f}_{21}^{T} & \ldots & \boldsymbol{f}_{24}^{T}
\end{array}\right]^{\mathrm{T}},
\end{aligned}
$$

with ${ }^{b} \boldsymbol{\eta}=\left[\begin{array}{ll}{ }^{b} \boldsymbol{\xi} & { }^{b} \dot{\xi}\end{array}\right]^{\mathrm{T}}$. Similarly, the forward kinematic actuation model (FKAM) can be derived from (6):

$$
\begin{gathered}
b \dot{\xi}=\boldsymbol{M}_{f}\left(\boldsymbol{\beta}_{m}\right) \dot{\boldsymbol{A}}_{b} \\
\boldsymbol{M}_{f}\left(\boldsymbol{\beta}_{m}\right)=\left[-d * \boldsymbol{F}_{2(d)}^{+}\left(\boldsymbol{\beta}_{m}\right) \quad r * \boldsymbol{F}_{2(d)}^{+}\left(\boldsymbol{\beta}_{m}\right)\right] .
\end{gathered}
$$

In (7), $\boldsymbol{\beta}$ is computed as a function of ${ }^{b} \dot{\boldsymbol{\xi}}$ using (4), whereas in (8) it is measured by the steer joint position sensor. Matrix $\boldsymbol{F}_{2(d)}^{+}=\left(\boldsymbol{F}_{2}^{\mathrm{T}} \boldsymbol{F}_{2}+\lambda^{2} \boldsymbol{I}_{3}\right)^{-1} \boldsymbol{F}_{2}^{\mathrm{T}}$ is the damped pseudo-inverse 
of $\boldsymbol{F}_{2}[15,16]$, with damping factor $\lambda$. Using it, the velocity trajectory can be reconstructed from joint velocity measurements, even at $\boldsymbol{F}_{2}$ singularities, making (8) a robust odometry model. Typically when moving with $\dot{\theta}=0$; such motion will yield a column of zeros depending on the direction of motion according to (7).

As mentioned earlier, mobile robots with steerable wheels can perform complex planar trajectories, provided their wheels orient to the proper initial values. These initial steer angles depend on the trajectory to be performed, and can be obtained, using (4) as:

$$
\beta_{i(\text { init })}=\tan ^{-1}\left(\frac{{ }^{b} \dot{y}_{\left(t_{\text {init }}+T_{s}\right)}+h_{x i} \dot{\theta}_{\left(t_{\text {init }}+T_{s}\right)}}{{ }^{b} \dot{x}_{\left(t_{\text {init }}+T_{s}\right)}-h_{y i} \dot{\theta}_{\left(t_{\text {init }}+T_{s}\right)}}\right) .
$$

In (9), $T_{s}$ is the sample time and the subscript $\left(t_{\text {init }}+T_{S}\right)$ denotes the value of the desired velocity component at the first sample time of the trajectory. Since the time needed to initialize the steer joints must be taken into account in the motion planning phase, it will affect the desired trajectory timing. To minimize such effect, the initial steer angles must be attained as fast as possible. In addition to using the maximum joint acceleration and/or velocity limits, we apply the following modification on the output of (9), to minimize the motion, based on the current configuration $\beta_{i(c)}$ :

$$
\beta_{i(\text { init })}=\left\{\begin{aligned}
\beta_{i(\text { init })}-\pi, & \forall \beta_{i(\text { init })}-\beta_{i(c)}>\pi / 2 \\
\beta_{i(\text { init })}+\pi, & \forall \beta_{i(\text { init })}-\beta_{i(c)}<\pi / 2, \\
\beta_{i(c)}, & \forall \beta_{i(\text { init })}-\beta_{i(c)}=\pi
\end{aligned}\right.
$$

The formula provided in (10) guarantees that the initial steer joint angle will always be $\leq \pm \pi / 2$.

\section{SingUlARITY TREATMENT}

Singularities related to this kind of mobile robots are divided into two categories: kinematic and representational. While the latter (for 3 or more steerable WMR) is solved by using the Cartesian coordinates of the base frame [1], the former still presents a challenging problem. Kinematic singularity refers usually to the case where the ICR passes by (or nearby) any of the steering axes. In such case, the steer angle $\beta_{i}$ in not uniquely defined, as there exist infinite values respecting the kinematic constraints. While passing this configuration, the steering rate shall grow unbounded from the "nearby" to the "at" singular configuration. This is shown mathematically in (4), where the denominator of the argument of the tan inverse function decreases to zero at such singularity. In this paper, we fix this problem with a very simple, yet effective, numerical treatment, by adding the damping parameters $\delta_{1}$ and $\delta_{2}$ respectively to the denominator of the tan inverse argument for the steer angle:

$$
\beta_{i}=\tan ^{-1}\left(\frac{\tilde{y}_{i}}{\widetilde{x}_{i}+\delta_{1} * \operatorname{sign}\left(\widetilde{x}_{i}\right)}\right)
$$

and to the denominator of the partial derivatives in $\boldsymbol{f}_{1 i}$ :

$$
\begin{gathered}
\frac{\partial \beta_{i}}{\partial^{b} \dot{x}}=\frac{-\tilde{y}_{i}}{\tilde{x}_{i}^{2}+\tilde{y}_{i}^{2}+\delta_{2}}, \\
\frac{\partial \beta_{i}}{\partial^{b} \dot{y}}=\frac{\tilde{x}_{i}}{\tilde{x}_{i}^{2}+\tilde{y}_{i}^{2}+\delta_{2}}, \quad \frac{\partial \beta_{i}}{\partial \dot{\theta}}=\frac{h_{x i}{ }^{b} \dot{x}+h_{y i}{ }^{b} \dot{y}}{\tilde{x}_{i}^{2}+\tilde{y}_{i}^{2}+\delta_{2}}
\end{gathered}
$$

where $\tilde{y}_{i}={ }^{b} \dot{y}+h_{x i} \dot{\theta}, \quad \tilde{x}_{i}={ }^{b} \dot{x}-h_{y i} \dot{\theta}$. Although the developed inverse kinematic model aims at providing joint steer rates, rather than joint steer angles, it is important to provide a singularity-free model for the steer joint variable too, since this is used in initializing robot configuration at the start of each new trajectory via (9), which justifies the need for (4'). The value of $\delta_{1}$ can be arbitrarily adjusted to be infinitesimally small, while $\delta_{2}$ should respect the acceleration limits of the steer joints (in case of centered WMRs) or of the steer/drive joints (in case of off-centered WMRs). Such effect is shown in Fig. 3(b). Once tuned, $\delta_{2}$ provides a damping effect which is completely the opposite to the singular behavior. Using (5') the steering rate will decrease to zero from the "nearby" to the "at" singular configuration. In order to tune $\delta_{2}$ to respect steer acceleration limits, we differentiate ( $\left.5^{\prime}\right)$ with respect to time, neglecting higher order derivatives, to obtain:

$$
\ddot{\beta}_{i}=\frac{k\left({ }^{b} \dot{\xi}, b \ddot{\xi}\right)}{\left(\tilde{x}_{i}^{2}+\tilde{y}_{i}^{2}+\delta_{2}\right)^{2}},
$$

where $k\left({ }^{b} \dot{\xi}, b \ddot{\xi}\right)=2\left(\tilde{y}_{i} \tilde{\dot{x}}_{i}-\tilde{x}_{i} \tilde{\dot{y}}_{i}\right)\left(\tilde{x}_{i} \tilde{\dot{x}}_{i}+\tilde{y}_{i} \tilde{\dot{y}}_{i}\right)$ is a function of the base frame trajectory. We define a candidate value of $\delta_{2}\left(\right.$ called $\left.\delta_{2 c}\right)$ as:

$$
\delta_{2 c}=\sqrt{\frac{\left|k\left({ }^{b \dot{\xi}}, b \ddot{\xi}\right)\right|}{\ddot{\beta}_{i(\max )}}}-\left(\tilde{x}_{i}^{2}+\tilde{y}_{i}^{2}\right),
$$

where $\ddot{\beta}_{i(\max )}$ is the $i^{\text {th }}$ steer joint acceleration limit in centered steerable robots, or the smallest acceleration limit of the steer/drive joint in off-centered steerable robots. Although the value obtained in (12) depends on the trajectory, to respect the acceleration limits at all times, we can conservatively use, for $\delta_{2}$, the maximum value over all possible trajectories in a bounded domain around the singularity. This value exists, since $\delta_{2 c}$ is upper-bounded, in such a bounded domain around the singularity. In applications involving human-robot collaboration, the trajectory is generally unknown a priori. In this case, we need to evaluate the maximum $\delta_{2 c}$ online. For this, we use:

$$
\max \left(x_{k}\right)=\left\{\begin{aligned}
x_{k}, & x_{k}>x_{k-1} \\
x_{k-1}, & \text { otherwise }
\end{aligned}\right.
$$

which will store the input argument with highest magnitude, where $(*)_{k}$ and $(*)_{k-1}$ denote a value at the current and the previous sampling instances respectively. Also, (12) has shown empirically (the authors intend to prove this in the future) to evaluate to a positive value in the neighborhood of kinematic singularity. In this region of interest, where respecting the acceleration limits becomes critical, we set $\delta_{2}$ to $\max \left(\delta_{2 c}\right)$. We should point out that this is the minimum value that can be used in $\left(5^{\prime}\right)$, while respecting the joint acceleration limit. Indeed, we need the minimum value so as to obtain the highest accuracy in evaluating the steer rate command. Finally, the online evaluation of $\delta_{2}$ is done using:

$$
\delta_{2}=\left\{\begin{array}{rr}
\delta_{1} & \forall \delta_{2 c} \leq 0 \\
\max \left(\delta_{2 c}\right) & \forall \delta_{2 c}>0
\end{array} .\right.
$$


Thanks to (13), the smallest $\delta_{2}$ value can be obtained online and set only in the vicinity of singular configurations at which the acceleration limits are to be monitored. Away from the critical zone (around singularity), this formula provides negligibly small value $\left(\delta_{1}\right)$ for $\delta_{2}$, consequently much higher accuracy for $\left(5^{\prime}\right)$. In what follows we prove that using (13) guarantees respecting the joint acceleration limits:

Case I: $\delta_{2 c} \leq 0$

From (12), this case corresponds to:

$$
\sqrt{|k(b \dot{\xi}, b \ddot{\xi})| / \ddot{\beta}_{i(\max )}} \leq \tilde{x}_{i}^{2}+\tilde{y}_{i}^{2}
$$

and we can assume that:

$$
\tilde{x}_{i}^{2}+\tilde{y}_{i}^{2}=\sqrt{|k(b \dot{\xi}, b \ddot{\xi})| / \ddot{\beta}_{i(\max )}}+c
$$

where $c \in \mathbb{R}^{+}$(positive semidefinite). Substituting by (14) in (11) where $\delta_{2}=\delta_{1}$ we obtain:

$$
\ddot{\beta}_{i}=\frac{k(b \dot{\boldsymbol{\xi}}, b \ddot{\boldsymbol{\xi}})}{\frac{|k(b \dot{\boldsymbol{\xi}}, b \ddot{\boldsymbol{\xi}})|}{\ddot{\beta}_{i(\max )}}+2 c_{1} \sqrt{\frac{|k(b \dot{\boldsymbol{\xi}}, b \ddot{\boldsymbol{\xi}})|}{\ddot{\beta}_{i(\max )}}}+c_{1}^{2}},
$$

where $c_{1}=\left(c+\delta_{1}\right) \in \mathbb{R}^{+}$. Rearranging we obtain:

$$
\left|\frac{\ddot{\beta}_{i}}{\ddot{\beta}_{i(\max )}}\right|=\frac{\left|k\left({ }^{b} \dot{\xi}, b \ddot{\xi}\right)\right|}{\left|k\left({ }^{b} \dot{\xi}, b \ddot{\xi}\right)\right|+2 c_{1} \sqrt{\left|k\left({ }^{b} \dot{\xi}, b \ddot{\xi}\right)\right| \ddot{\beta}_{i(\max )}}+c_{1}^{2} \ddot{\beta}_{i(\max )}},
$$

From (16), it is evident that the right hand side will always be smaller than one. Consequently, the steer joint acceleration will comply with the maximum limit.

Case II: $\delta_{2 c}>0$

In this case, we assume that the value of $\delta_{2}$ is constant and as such (11) still holds true. This is a reasonable assumption after few movements of the robot passing by kinematic singularity, where it quickly converges to the maximum value of $\delta_{2 c}$. Then (12) becomes:

$$
\sqrt{\left|k\left({ }^{b} \dot{\xi}, b \ddot{\xi}\right)\right| / \ddot{\beta}_{i(\max )}}>\tilde{x}_{i}^{2}+\tilde{y}_{i}^{2}
$$

and we can assume that:

$$
\tilde{x}_{i}^{2}+\tilde{y}_{i}^{2}=\sqrt{|k(b \dot{\xi}, b \ddot{\xi})| / \ddot{\beta}_{i(\max )}}-c,
$$

where $c \in \mathbb{R}^{+}$. Substituting by (17) in (11), we obtain the same formulae in (15) and (16) with $c_{1}=\max \left(\delta_{2 c}\right)-c$. However the definition of the $\max (*)$ function guarantees that $\max \left(\delta_{2 c}\right) \geq c$, so $c_{1} \geq 0$ and consequently $\ddot{\beta}_{i} \leq$ $\ddot{\beta}_{i(\max )}$.

\section{BENCHMARK TESTING TRAJECTORY}

In this section, we propose a velocity trajectory to benchmark test the model and controller performances w.r.t representational and kinematic singularities. The benchmark

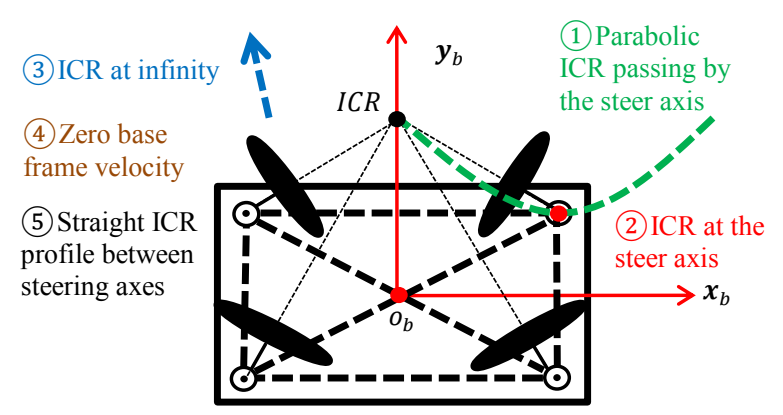

Fig. 2 ICR position in the base frame in each of the five motion tests of the proposed benchmark testing trajectory.

is composed of five motion profiles (all defined in the robot frame), depicted in Fig. 2:

1) ICR approaching, coinciding with and departing from a steering axis. We employ a parabolic ICR motion profile with vertex at the steering axis (note that a straight line would impose no motion on the steering joint).

2) ICR coincident with a steering axis: the robot pivots about that axis.

3) Straight line motion in the direction of one of the axes (ICR at infinity). This tests the proposed singularity avoidance algorithm for zero angular velocity, i.e., at a representational singularity of ICR-based kinematic models. It also tests the developed forward kinematic model, since it will lead to a column of zeros, hence a rank loss in $\boldsymbol{F}_{2}$.

4) Zero linear and angular velocities ${ }^{b} \dot{\xi}=\left[\begin{array}{lll}0 & 0 & 0\end{array}\right]^{T}$, which result in joint angles being undefined, refer to (4).

5) Straight line motion profile between two of each of the steering axes (i.e., six straight lines for a 4-steerable WMR). This motion profile tests the performance of kinematic models developed using only two steerable wheels, since then it will result in undefined steer joint values.

Since in our model development, we use four steerable wheels, we will exclude the fifth test from our benchmark trajectory. The results of numerical simulations (in $\mathrm{C}++$ ) of trajectories 1-4 are shown in Fig. 3.a, where $\ddot{\beta}_{i(\max )}$ is set to $5 \mathrm{rad} / \mathrm{s}^{2}$. To ensure a smooth behavior of each motion test, a linear trajectory with $5^{\text {th }}$ order blends (indicated in the subscript) is employed:

$T_{l 5 b}=\left\{\begin{aligned} a_{0}+a_{1} \Delta t_{1}+a_{2} \Delta t_{1}^{2}+a_{3} \Delta t_{1}^{3}+a_{4} \Delta t_{1}^{4}+a_{5} \Delta t_{1}^{5} & \forall t_{i} \leq t<t_{1} \\ a_{6}+a_{7} \Delta t_{2} & \forall t_{1} \leq t<t_{2} . \\ a_{0}+a_{1} \Delta t_{3}+a_{2} \Delta t_{3}^{2}+a_{3} \Delta t_{3}^{3}+a_{4} \Delta t_{3}^{4}+a_{5} \Delta t_{3}^{5} & \forall t_{2} \leq t<t_{f}\end{aligned}\right.$

Constants $a_{0 \rightarrow 7}$ in (18) depend on initial and terminal conditions, $t_{i}\left(t_{f}\right)$ denotes initial (final) time, and $\Delta t_{1}=$ $t-t_{i}, \Delta t_{2}=t-t_{1}, \Delta t_{3}=t-t_{2}$, while $t_{1}=t_{i}+0.1 *$ $\left(t_{f}-t_{i}\right)$ and $t_{2}=t_{f}-0.1 *\left(t_{f}-t_{i}\right)$.

For motion test $1, t=[4,8[$, we apply rigid body mechanics: the robot linear tangential velocity $v_{b}=R \dot{\theta}$, with $R$ the radius of curvature. For $\dot{\theta}=-1 \mathrm{rad} / \mathrm{s}$ (i.e., $\left.v_{b}=-R\right)$, it can be shown that: ${ }^{b} \dot{x}=-v_{b} \sin (\gamma)=$ $R \sin (\gamma)={ }^{b} y_{I C R}$ and ${ }^{b} \dot{y}=-{ }^{b} x_{I C R}$. The base trajectory can be expressed as:

$$
\begin{gathered}
{ }^{b} \dot{y}=T_{l 5 b}\left({ }^{b} \dot{y}_{i}=0,{ }^{b} \dot{y}_{f}=2 h_{x 2}, \quad t_{i}=4, t_{f}=8\right) \\
{ }^{b} \dot{x}=-\left({ }^{b} \dot{y}-h_{x 2}\right)^{2}-h_{y 2}, \quad \dot{\theta}=-1 .
\end{gathered}
$$



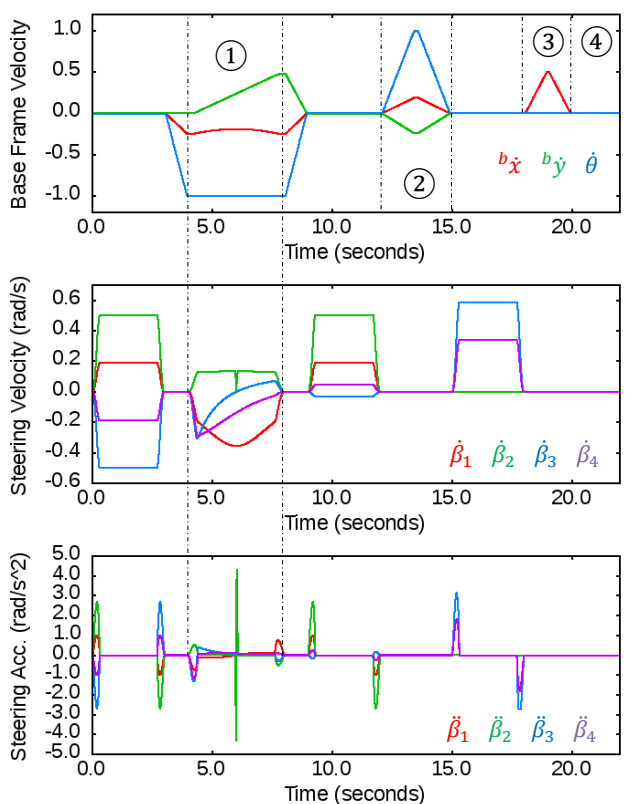

(a)

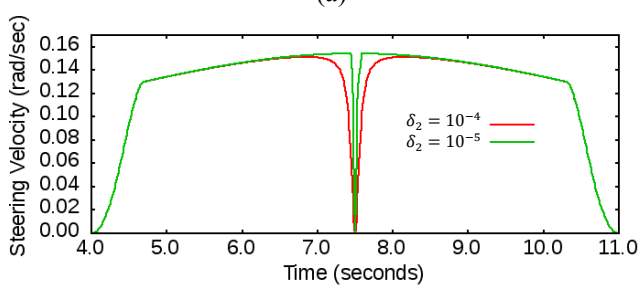

(b)

Fig. 3 Simulation results of the proposed numeric treatment; (a) base frame velocity trajectory ${ }^{b} \dot{\xi}$ of the first four benchmark tests (top), the corresponding steer joint rate commands (middle) and steer joint acceleration (limit of $5 \mathrm{rad} / \mathrm{s}^{2}$ ), (b) the effect on the second steer joint rate $\dot{\beta}_{2}$ passing kinematic singularity along with the steer rate variation with $\delta_{2}$.

Equation (19) gives a parabolic motion profile for ${ }^{b} \dot{x}$, with vertex at the $2^{\text {nd }}$ steering axis, while ${ }^{b} \dot{y}$ changes from 0 to $2 h_{x 2} \mathrm{~m} / \mathrm{s}$ in 4 seconds, following a linear trajectory with $5^{\text {th }}$ order blends. In test $2, t=[12,15$ [, the robot pivots about the $2^{\text {nd }}$ steer axis. It accelerates in $1.5 s$ and then decelerates in $1.5 \mathrm{~s}$. Test 3: $t=[18,20[$, imposes robot motion in $\mathrm{x}-$ axis; accelerating in $1 s$ and then decelerating in $1 \mathrm{~s}$. Finally, null robot velocity command is sent for $2 s$ to conclude test 4. Intervals $t=[0,3[, t=[9,12[$ and $t=[15,18[$ are dedicated to initialize the steer joints to the proper values, to start the corresponding trajectory. During intervals $t=[3,4[$ and $t=[8,9[$, the velocity respectively increases from null to the initial value of test 1 , and decreases from the final value of test 1 , to null. Steer joint rates for such trajectory with the proposed numeric treatment are shown in Fig. 3.a (middle). Damping effect of $\delta_{2}$ is shown in Fig. 3.b.

\section{EXPERIMENTS AND DISCUSSION}

In this section, we present the experiments conducted on the Neobotix MPO700 mobile robot to assess the proposed model, compare it with the embedded controller. The experiments are shown in Fig. 4, and in the video attached to

\footnotetext{
${ }^{1}$ www.coppeliarobotics.com/

2 https://www.youtube.com/watch?v=9RCOUp24Gvs
}

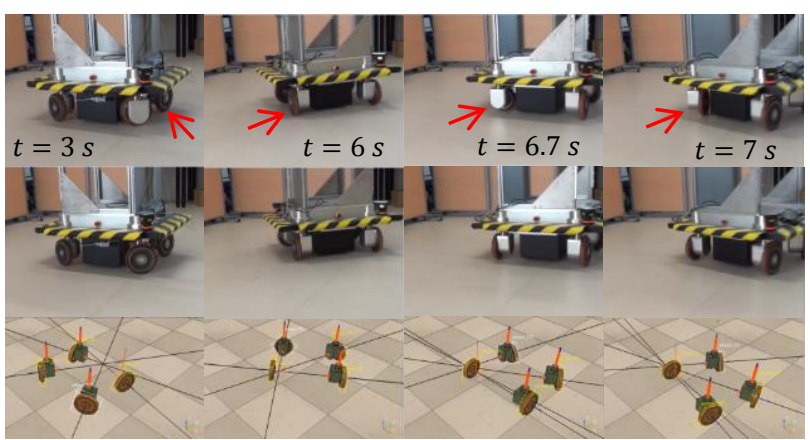

Fig. 4 Snapshots of the two experiments (test 1, with singular joint indicated by the red arrow). Top: embedded controller, Middle: developed model. Bottom: v-rep ${ }^{1}$ simulation showing the ICR position.

this paper (available online at: IDH-LIRMM youtube channel $^{2}$ ). The robot driver allows two modes of commands; joint velocity or robot velocity modes. We use the former to test the proposed model while the latter (employing the embedded model) is used in the comparative study. In a first experiment, the testing trajectory shown in Fig. 3.a (top) is sent in robot velocity mode, to evaluate the joint commands output by the embedded model. In a second experiment, the output of our inverse kinematic model shown in Fig. 3.a (middle) is used to control the robot in joint velocity mode.

Fig. 5.a and Fig .5.b respectively show the results for the embedded and developed models. For test 1 (parabolic motion of the ICR), the embedded controller gives a velocity peak for the steering joint in the neighborhood of the kinematic singularity, green curve, at $6.3 s$ on Fig. 5.a (top). This corresponds to a steer angle change of 180 degrees in a very short time (see Fig. 4 (top) at $t=6 \mathrm{~s}$ and $\mathrm{t}=7 \mathrm{~s}$ ), implying that the embedded model probably employs steer position, rather than steer velocity control. Corresponding wheel velocity response can be seen in Fig. 5.a (middle) and the effect of such inconvenient velocity command on the velocity error is evident in Fig. 5.a (bottom). The other undesirable - steering velocity peaks in Fig. 5.a (top) are due to the fact that the embedded controller goes to each initial trajectory configuration as quickly as possible.

The effect of the proposed model on the steering joint velocity is shown in Fig. 5.b (top). As shown, when approaching kinematic singularity, the velocity of the singular steer joint slows down to $0 \mathrm{rad} / \mathrm{s}$ at the kinematic singularity, with damped steer/drive rates in vicinity (observe Fig. 4 (middle) between $t=6 \mathrm{~s}$ and $\mathrm{t}=7 \mathrm{~s}$ ). Such effect is favorable, as it makes all the velocity space of the robot accessible, with minimum power consumption and with no risk of damage to motors performing at their top limits. Such damped behavior in the steering (Fig. 5.b, top) and driving (Fig. 5.b, middle) results in much smaller velocity error w.r.t the desired trajectory (Fig. 5.b, bottom). Both models show satisfactory response in the 3 other tests and as such the response is omitted from Fig. 4.

\section{CONCLUSION}

A complete kinematic model for steerable wheeled mobile robots has been presented in terms of forward (odometry), 

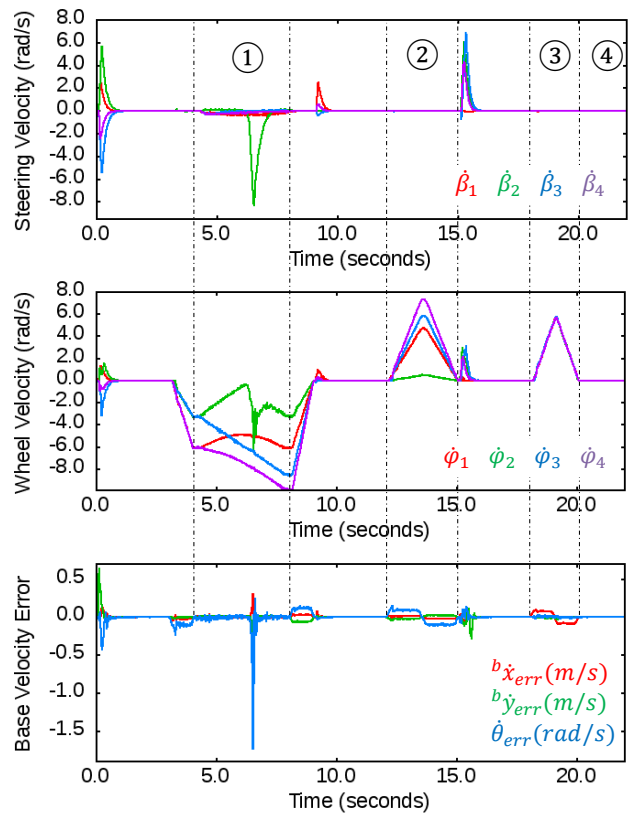

(a)
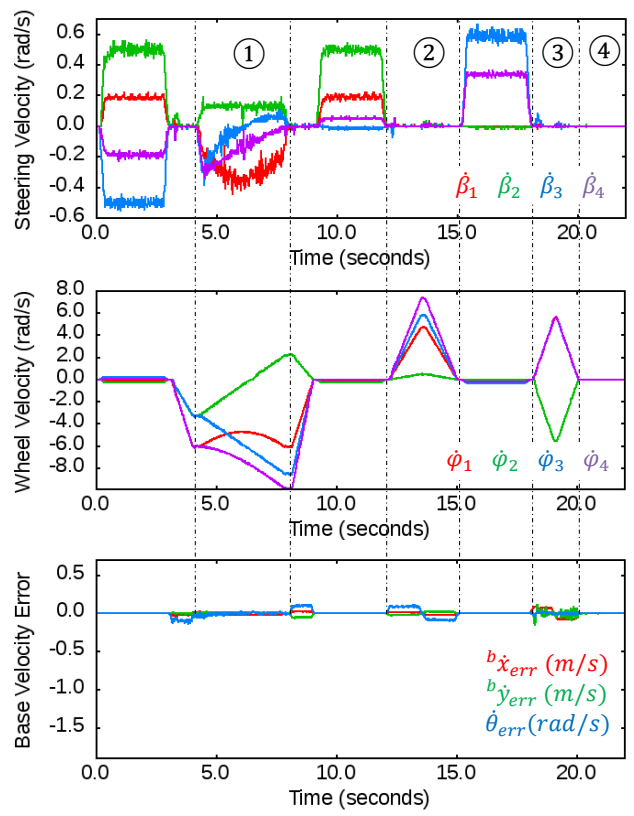

(b)

Fig. 5 Results of the benchmark test on the MPO700 employing (a) the embedded controller and (b) the proposed method. Each figure shows the steering joint velocity (top), the drive joint velocity (middle) and the error in velocity trajectory ( ${ }^{b} \dot{\xi}^{*}-{ }^{b} \dot{\boldsymbol{\xi}}_{r}$ with ${ }^{b} \dot{\boldsymbol{\xi}}^{*},{ }^{b} \dot{\xi}_{r}$ the desired and actual velocities) (bottom).

inverse (actuation) kinematics, and minimum steer angle initialization models. The inverse kinematic model presented is free of representational singularities. Additionally, a numeric treatment is provided to solve the kinematic singularity, hence providing a completely singular-free model. A benchmark velocity trajectory is proposed, to evaluate the performance of the developed model in all singular conditions that can be encountered by such systems. Using the proposed benchmark test, the developed model has been tested on the Neobotix MPO700 robot, and shown superior results as compared with the embedded controller.

\section{ACKNOWLEDGMENTS}

This work has been supported by the French region Languedoc Roussillon Midi Pyrénées (project CoBot@LR), by the PSA robotics department, and by French - German PHC Procope project ViNavHuBot.

\section{REFERENCES}

[1] A. Dietrich, T. Wimbock, A. Albu-Schaffer and G. Hirzinger, "Singularity avoidance for nonholonomic omnidirectional wheeled mobile platforms with variable footprint," in Proc. of the IEEE Int. Conf. on Robotics and Automation (ICRA), pp. 6136-6141, 2011.

[2] C. Connette, A. Pott, M. Hagele and A. Verl, "Control of an pseudoomnidirectional nonholonomic mobile robot based on an ICM representation in spherical coordinates," in Proc. of the $47^{\text {th }}$ IEEE Conf. on Decision nd Control (IDC), pp. 4976-4983, 2008.

[3] B. Thuilot, B. D'Andrea-Novel and A. Micaelli, "Modeling and feedback control of mobile robots equipped with several steering wheels," in IEEE Transactions on Robotics and Automation, vol.12, no.3, pp. 375-390, Nov 1996.

[4] U. Schwesinger, C. Pradalier and R. Siegwart, "A novel approach for steeringwheel synchronization with velocity/acceleration limits and mechanical constraints," in Proc. of the IEEE Int. Conf. on Inteligent Robots and Systems (IROS), pp. 5360-5366, 2012.

[5] P. R. Giordano, M. Fuchs, A. Albu-Schaffer and G. Hirzinger, "On the kinematic modeling and control of a mobile platform equipped with steering wheels and movable legs," in Proc. of the IEEE Int Conf. on Robotics and Automation (ICRA), pp. 4080-4087, 2009.

[6] A. Betourne, and G. Campion, "Kinematic modeling of a class of omnidirectional mobile robots," in Proc. of the IEEE Int. Conf. on Robotics and Automation (ICRA), pp. 3631-3636, 1996.

[7] C. Connette, C. Parlitz, M. Hagele and A. Verl, "Singularity avoidance for overactuated pseudo-omnidirectional wheeled mobile robots," in Proc. of the IEEE Int. Conf. on Robotics and Automation (ICRA), pp. 4124-4130, 2009

[8] R. Oftadeh, R. Ghabcheloo and J. Mattila, "Time optimal path following with bounded velocities and accelerations for mobile robots with independently steerable wheels," in Proc. of the IEEE Int. Conf. on Robotics and Automation (ICRA), pp. 2925-2931, 2014.

[9] S. Chamberland, E. Beaudry, L. Clavien, F. Kabanza, F. Michaud and M. Lauria, "Motion planning for an omnidirectional robot with steering constraints," in Proc. of the IEEE Int. Conf. on Inteligent Robots and Systems (IROS), pp. 4305-4310, 2010.

[10] C. Connette, M. Hagele and A. Verl, "Singularity-free state-space representaion for non-holonomic omnidirectional undercarriages by means of coordinate switching," in Proc. of the IEEE Int. Conf. on Inteligent Robots and Systems (IROS), pp. 4959-4965, 2012.

[11] R. Oftadeh, R. Ghabcheloo and J. Mattila, "A novel time optimal path following controller with bounded velocities for mobile robots with independently steerable wheels," in Proc. of the IEEE Int. Conf. on Inteligent Robots and Systems (IROS), pp. 4845-4851, 2013.

[12] P. Muir, and C. Neumann, "Kinematic modeling of wheeled mobile robots," Journal of Robotic Systems, vol. 4, no. 2, pp. 282-340, 1987.

[13] G. Campion, G. Bastin, and B. D'Andrea-Novel, "Structural properties and classification of kinematic and dynamic models of wheeled mobile robots," in IEEE Trans. on Robotics and Automation, pp. 3631-3636, 1996.

[14] K. Low, and Y. Leow, "Kinematic modeling, mobility analysis and design of wheeled mobile robots," in Advanced Robotics, vol.19, no.1, pp. 73-99, 2005.

[15] Y. Nakamura a nd H. Hanafusa, "Inverse kinematic solutions with singularity robustness for robot manipulator control," in Trans. of ASME J. of Dynamic Systems, Measurement, and Control, vol. 108, pp. $163-171,1986$

[16] C. Wampler, "Manipulator inverse kinematic solutions based on vector formulations and damped least-squares methods," in IEEE Trans. on Systems, Man, and Cybernetics, vol. 16, pp. 93-101, 1986. 